\title{
Application of High Hydrostatic Pressure for Production of Bioactive Soyasaponin from Black Soybean [Glycine max (L.)] Sprout
}

\author{
Min Young Kim', Gwi Yeong Jang ${ }^{2}$, Yoon Jeong Lee ${ }^{3}$, Kyung Mi Kim4, \\ Dae Joong Kim ${ }^{5}$ Junsoo Lee ${ }^{3}$, Heon Sang Jeong,**

\begin{abstract}
${ }^{1}$ Department of Central Area Crop Science, National Institute of Crop Science, Rural Development Administration, Suwon, 16429, South Korea
${ }^{2}$ Department of Herbal Crop Research, National Institute of Horiticultural and Herbal Science, Rural Development Administration, Eumseong 27709, South Korea

${ }^{3}$ Depatment of Food Science and Biotechnology, Chungbuk National University, Cheongju 28644, South Korea

${ }^{4}$ National Academy of Agricultural Science, Rural Development Administration, Jeonju 54875, Korea

${ }^{5}$ Veterinary Medical Center and College of Veterinary Medicine, Chungbuk National University, Cheongju 28644, South Korea

*Corresponding author: hsjeong@chungbuk.ac.kr
\end{abstract}

Received January 10, 2019; Revised March 04, 2019; Accepted April 01, 2019

\begin{abstract}
This investigation aims to evaluate the influence of high hydrostatic pressure (HHP) treatment after germination on soyasaponin conversion and physiological activity in black soybean [Glycine max (L.)]. Black soybean was germinated for 4 days and subjected to $24 \mathrm{~h}$ at 0.1-150 MPa; the crude soyasaponin extracts were then evaluated for anti-inflammatory and anti-obesity activity. The highest crude soyasaponin content of $57.85 \mathrm{mg} / \mathrm{g}$ was found in germinated black soybean treated at $100 \mathrm{MPa}$. Soyasaponin Bb and Bc were the major B-group soyasaponins in raw black soybean; their contents in raw and germinated black soybean increased after HHP treatment. The lipid accumulation rate during 3T3-L1 adipocyte differentiation in germinated HHP-treated black soybean ranged from $93.25-65.95 \%$ at the concentration of $400 \mu \mathrm{g} / \mathrm{mL}$. The highest anti-obesity effect was observed after germination and 150-MPa treatment. Black soybean sprout extracts treated at $150 \mathrm{MPa}$ significantly inhibited the lipopolysaccharide-induced expression of inflammatory markers by RAW 264.7 macrophages.
\end{abstract}

Keywords: black soybean sprout, high hydrostatic pressure, soyasaponin, anti-inflammatory activity, anti-obesity activity

Cite This Article: Min Young Kim, Gwi Yeong Jang, Yoon Jeong Lee, Kyung Mi Kim, Dae Joong Kim, Junsoo Lee, and Heon Sang Jeong, "Application of High Hydrostatic Pressure for Production of Bioactive Soyasaponin from Black Soybean [Glycine max (L.)] Sprout.” Journal of Food and Nutrition Research, vol. 7, no. 4 (2019): 270-278. doi: 10.12691/jfnr-7-4-3.

\section{Introduction}

Soyasaponins belong to amphiphilic oleanane triterpenoid glycosides of aglycone with one or two polysaccharide chains. Differences in the aglycone compounds permit classification of soyasaponins as groups A, B, or E [1]. Soyasaponins have been reported to exert antioxidative [2,3], cholesterol-lowering [4], renin-inhibiting [5], hepatoprotective [6], and antitumor effects [7]. Most of these effects have been revealed using single compounds of group B soyasaponin; the conversion characteristics and biological activities of crude soyasaponin extracts with various treatments remain unclear.

Legumes could provide ideal nutritional components such as carbohydrates (dietary fibre), protein, fatty acids, vitamins and minerals complementary to cereal-based diets [8]. In addition, the abundant phytochemicals including soyasaponin in legumes are known to have various physiological activities such as antioxidant, anti-inflammatory and anti-proliferation abilities [9]. Black soybeans are a widely used health food, and an important source of oil, protein, and secondary metabolites including soyasaponin [10]. Among various soybean and black soybean products, sprouts, which are rich in dietary fiber, various nutrients, and bioactive components, are the valuable dietary supplements in many parts of the world that may promote health and well-being [11]. Germination is considered as an inexpensive and effective technology to improve the nutritional quality of soybean because this process triggers a sequence of metabolic changes [12]. Along with saponins and isoflavones change during germination because of accumulation and synthesis [12]. These changes in composition enhance the overall nutritional value of the soybeans and the contents of health-promoting phytochemicals [13].

High-pressure technology is used in an increasing variety of fields related to biology, and as a non-thermal food processing technique as an alternative to high-heat 
processing in the food industry [14]. The use of high hydrostatic pressure (HHP) has gained prominence as a tool to perturb biochemical systems to establish the relationships between molecular structures and functions [15]. In a previous study, Kim et al. [16] reported the application of HHP technology to black soybeans to enhance their production of bioactive peptides related to inflammation makers.

The beneficial effects of soyasaponins for the reduction in incidence of chronic diseases have been linked to their modulation of obesity and inflammation factor, and the single processing methods of germination and HHP have been investigated to efficaciously enhance the functional characteristics of legumes. However, this is the first study about evaluation of physiological properties in soyasaponin rich fraction produced using high hydrostatic pressure treatment of black soybean sprout. The present study investigates the improvement of the anti-inflammatory and anti-obesity characteristics of crude soyasaponins extracted from black soybean by the combined effects of germination and HHP treatment.

\section{Materials and Methods}

\subsection{Preparation of Black Soybean Sprout}

Black soybean [Glycine max (L.) Merr.] were macerated in distilled water (seed:water proportion of 1:5, w/v) at $20^{\circ} \mathrm{C} \pm 1^{\circ} \mathrm{C}$ for $24 \mathrm{~h}$. The water used for macerating was dehydrated and changed every $3 \mathrm{~h}$. The macerated seeds were laid down a germination tray $(100 \times 150 \mathrm{~mm})$ comprising wet laboratory cotton stuff. They were then covered with another layer of wet cotton stuff and placed in a seed germinator (WGC 450, Dahan, Seoul, Korea) in contact with circulating water, ensuring that the seeds were constantly wet through capillary action. The seeds were left in darkness at $20^{\circ} \mathrm{C}$ and $95 \%$ relative humidity (RH) for 4 days [17]. The optimum germination period of 4 days was selected according to a preliminary experiment on the functional compounds and physiological activities of black soybean with germination periods varying from 0 to 6 days (data not shown).

\subsection{High Hydrostatic Pressure (HHP) Treatment}

The germinated black soybean was carried out HHP operating a warm isostatic press pressure-treatment system (WIP-L60-50-200, Ilshin Autoclave Co., Ltd., Daejeon, Korea), with the pressure chamber maintained at room temperature $\left(\mathrm{RT}, 20^{\circ} \mathrm{C}\right)$. The warm isostatic press applied isostatic pressure using water as the pressure medium with neither heat nor gas. The apparatus are composed of reservoir tank, high-pressure pump and vessel, control system, alarm system and safety device. Samples (20 g) of macerated black soybean and 4-day germinated black soybean were blended with $20 \mathrm{~mL}$ of distilled water. The mixtures were transferred to a laminated aluminum foil film (Newpack, Seoul, Korea) and heat-sealed using vacuum packaging (chamber-type vacuum package, DP-901, Dew Pack Korea Machinery Co., Seoul, Korea). HHP treatment was performed immediately after germination to prevent enzyme inactivation. The packaged samples were subjected to $0.1,50,100$, or $150 \mathrm{MPa}$ at $25^{\circ} \mathrm{C}$ for $24 \mathrm{~h}$. All samples were dried using a freezing drier and stored at $-20^{\circ} \mathrm{C}$ in a deep freezer (Ultra-low Temperature Freezer, MDF-393, SANYO, Akaiwa, Japan).

\subsection{Extraction of Crude Soyasaponin}

Soyasaponins were extracted according to the method of Berhow et al. [18] with slight modifications. Briefly, the samples were ground using a hammer mill and then the 100-mesh powdered samples were defatted three times with hexane at $25^{\circ} \mathrm{C}$ for $1 \mathrm{~h}$ using a shaking incubator before the extraction of crude soyasaponin. The defatted samples were extracted three times with $80 \%$ ethanol at RT for $1 \mathrm{~h}$ using an ultrasonic bath (SD-350H; Seong Dong, Seoul, Korea). The extracts were filtered, combined, and concentrated using a rotary evaporator under vacuum. The residue was dissolved in distilled water and extracted three times with water-saturated n-butyl alcohol. The nbutyl alcohol layer was evaporated using a rotary evaporator under vacuum and dissolved in distilled water. The dissolved extract was dried using a freeze dryer (FD5508; Ilshin BioBase, Yangju, Korea), and the dried extract was used to determine the crude soyasaponin content (mg/g soybean).

\subsection{Analysis of Soyasaponin Composition}

The soyasaponin composition of each extract was determined using a high-performance liquid chromatography (HPLC) system according to the method described by Hubert, Berger, and Dayde [19] with slight modifications. Each crude soyasaponin extract was dissolved in methanol and filtered through a $0.45-\mu \mathrm{m}$ syringe filter (Millipore, Billerica, MA, USA). The analytical column was an octadecylsilyl (ODS) column $(5 \mu \mathrm{m}, 4.6 \times 250 \mathrm{~mm}$, Agilent Technologies). A gradient elution was employed using solvent A (water containing $0.025 \%$ (v/v) trifluoracetic acid) and solvent B (acetonitrile containing $0.025 \%(\mathrm{v} / \mathrm{v})$ trifluoracetic acid). The gradient program was as follows: $0-55 \mathrm{~min}, 70 \%$ to $40 \% \mathrm{~A}$ in B (gradient); 55-56 min, $40 \%$ to $0 \% \mathrm{~A}$ in $\mathrm{B}$ (gradient); 56-65 min, 0\% to $0 \% \mathrm{~A}$ in $\mathrm{B}$ (gradient); $65-66 \mathrm{~min}, 0 \%$ to $70 \% \mathrm{~A}$ in $\mathrm{B}$ (gradient); 66-75 min, 70\% $\mathrm{A}$ in $\mathrm{B}$ (isocratic). The flow rate was kept at $1 \mathrm{~mL} / \mathrm{min}$ and the injection volume was $20 \mu \mathrm{L}$. The UV detector was set at $205 \mathrm{~nm}$. A soyasaponin standard mixture, containing soyasaponins $\mathrm{Aa}, \mathrm{Ab}, \mathrm{Ba}, \mathrm{Bb}$ and $\mathrm{Bc}$ and soyasapogenols $\mathrm{A}$ and $\mathrm{B}$, was prepared in HPLC-grade methanol. The soyasaponin concentrations were determined by standard curves obtained by injecting different concentrations of the soyasaponin standard into the HPLC system. Peaks were verified by adding standard phenolic acids to the samples, and each peak area was calculated relative to a standard peak area. The total soyasaponin content was calculated by adding up the different soyasaponin component amounts.

\subsection{Cell Culture and MTT Assay for Cell Viability}

RAW 264.7 cells and 3T3-L1 pre-adipocytes were obtained from the Korean Cell Line Bank (Seoul, Korea) and 
American Type Culture Collection (Manassas, VA, USA) respectively. RAW 264.7 cells and 3T3-L1 pre-adipocytes were maintained in separate incubators containing Dulbecco's modified Eagle's medium (DMEM) with 10\% fetal bovine serum (FBS), $100 \mathrm{U} / \mathrm{mL}$ penicillin, and 50 $\mu \mathrm{g} / \mathrm{mL}$ streptomycin at $37^{\circ} \mathrm{C}$ with $5 \% \mathrm{CO}_{2}$. RAW264.7 cell and 3T3-L1 pre-adipocyte death was measured using a 3-(4,5-dimethylthiazol-2-yl)-2,5-diphenyltetrazolium bromide (MTT) assay [20]. RAW 264.7 and 3T3-L1 pre-adipocytes were seeded at $5 \times 10^{4}$ cells/well in $100 \mu \mathrm{L}$ in 96-well plates and incubated for $24 \mathrm{~h}$ to allow adherence, then treated with different concentrations of soyasaponin extracts $(50,100,200$, and $400 \mu \mathrm{g} / \mathrm{mL})$. After incubation for $24 \mathrm{~h}$, a $10-\mu \mathrm{L}$ aliquot of $2 \mathrm{mg} / \mathrm{mL}$ MTT reagent was added to each well, and the plates were incubated at $37^{\circ} \mathrm{C}$ in $5 \% \mathrm{CO}_{2}$ humidified air for $2 \mathrm{~h}$. The supernatant was carefully removed and $100 \mu \mathrm{L}$ of dimethyl sulfoxide (DMSO) was added to each well. The resulting color was evaluated at $540 \mathrm{~nm}$ using an ELISA microplate reader (ELx808, Bio-Tek®, Winooski, VT, USA). Cytotoxicity was calculated as a percentage of control cell viability.

\subsection{Measurement of Nitric Oxide (NO) Production}

The measurement of nitrite using Griess reagents is widely utilized to indirectly evaluate NO production. Nitrite is a relatively stable $\mathrm{NO}$ oxidation product in conditioned media and can be measured by spectrophotometry [21]. Briefly, RAW 264.7 cells $\left(5 \times 10^{4}\right.$ cells/well) were seeded in 96-well plates and incubated for $6 \mathrm{~h}$ at $37^{\circ} \mathrm{C}$. The cells were treated with or without $0.5 \mu \mathrm{g} / \mathrm{mL}$ lipopolysaccharides (LPS) and the indicated concentrations of soybean protein extracts for $24 \mathrm{~h}$. Then, the concentration of NO in the medium was measured using a Griess Reagent System (Promega, Madison, WI, USA). The absorbance was read at $550 \mathrm{~nm}$ on a microplate reader. The NO concentration was determined by comparison to dilutions of sodium nitrite as a standard.

\subsection{Quantification of Pro-inflammatory Cytokine Production}

RAW 264.7 cells $\left(5 \times 10^{4}\right.$ cells/well $)$ were seeded in 96-well plates and incubated for $6 \mathrm{~h}$ at $37^{\circ} \mathrm{C}$. Cells were treated with or without $0.5 \mu \mathrm{g} / \mathrm{mL}$ LPS and the indicated concentrations of soybean protein extracts for $24 \mathrm{~h}$. After $24 \mathrm{~h}$ of treatment, the culture supernatants were collected. Tumor necrosis factor $\alpha$ (TNF- $\alpha)$, interleukin (IL)-1 $\beta$, and IL-6 were measured using $\operatorname{Cymax}^{\mathrm{TM}}$ Mouse enzyme-linked immunosorbent assay (ELISA) kits (Abfrontier, Seoul, Korea), according to the instructions provided by the manufacturer.

\subsection{Adipocyte Differentiation and Oil Red O Staining}

The 3T3-L1 cells were grown in DMEM containing $10 \%$ FBS, $100 \mathrm{U} / \mathrm{mL}$ penicillin, and $50 \mu \mathrm{g} / \mathrm{mL}$ streptomycin at $37^{\circ} \mathrm{C}$ in an incubator with $5 \% \mathrm{CO}_{2}$ until confluent. For adipocyte differentiation, the cells were cultured into 24 -well plates at a density of $1 \times 10^{5}$ cells $/ \mathrm{mL}$, and 2-day post-confluent pre-adipocytes were stimulated for $48 \mathrm{~h}$ with DMEM containing 10\% FBS and a hormone cocktail (0.5 mM-isobutyl-3-methylxanthine, $1 \mu \mathrm{M}$-dexamethasone, $5 \mu \mathrm{g} / \mathrm{mL}$ insulin). Cells were then cultured in DMEM supplemented with 10\% FBS and $5 \mu \mathrm{g} / \mathrm{mL}$ insulin for another 2 days and were maintained with 10\% FBS plus DMEM for an additional 2 days. To test the effect of samples on adipogenesis, the differentiation of 2-day post-confluent pre-adipocytes was induced in the presence or absence of the test sample for 4 days.

On day 6, the mature adipocytes were subjected to Oil Red $\mathrm{O}$ staining. After removal of the culture medium, the cells were fixed with $10 \%$ formaldehyde in phosphate-buffered saline (PBS) for $1 \mathrm{~h}$ at room temperature and were then washed twice with PBS. The fixed cells were stained with Oil Red O staining solution (0.3\% Oil Red O in 60\% isopropyl alcohol) for $20 \mathrm{~min}$, followed by two washes in PBS. The stained lipid droplets were photographed using a microscope (Carl Zeiss, Jena, Germany). Finally, the dye retained in the cells was dissolved in isopropyl alcohol and was quantified by measuring the absorbance at $520 \mathrm{~nm}$ using a microplate reader. All samples were evaluated in triplicate and results were reported as mean \pm standard deviation.

\subsection{Statistical Analysis}

Results are reported as mean \pm standard deviation $(n=3)$. The statistical significance of differences among treatments was determined using the one-way analysis of variance (ANOVA) calculated by SPSS version 12 (SPSS Institute, Chicago, IL, USA) with a significance level of $P<0.05$ determined by the Duncan multiple range test.

\section{Results and Discussion}

\subsection{Crude Soyasaponin Content}

The Influence on germination and HHP treatment of the crude soyasaponin contents in black soybean is shown in Figure 1. High hydrostatic pressure treatment after germination resulted in obvious enhanced effects on the crude soyasaponin contents of black soybean. The black soybean sprout presented $50.76 \mathrm{mg} . / \mathrm{g}$ of cude soyasaponin in average, which was significantly higher than raw black soybean about $39.36 \mathrm{mg} / \mathrm{g}$. Similarly, Chang and Han [22] observed a greatly increased enhanced the crude soyasaponin contents in general soybean cultivars applying germination, although the crude soyasaponin contents were slightly decreased during soaking processes. These increases in soyasaponins demonstrated that soyasaponins were not only were generated by biosynthetic pathways with enzyme activation as secondary metabolites, but also increased the activity of cell-wall hydrolytic enzyme and the extraction yield of soyasaponins in black soybean [23,24]. In other words, the germination effects on soyasaponin contents are based on the biosynthesis or conversion of soyasaponin to create secondary metabolites essential for plant growth. 


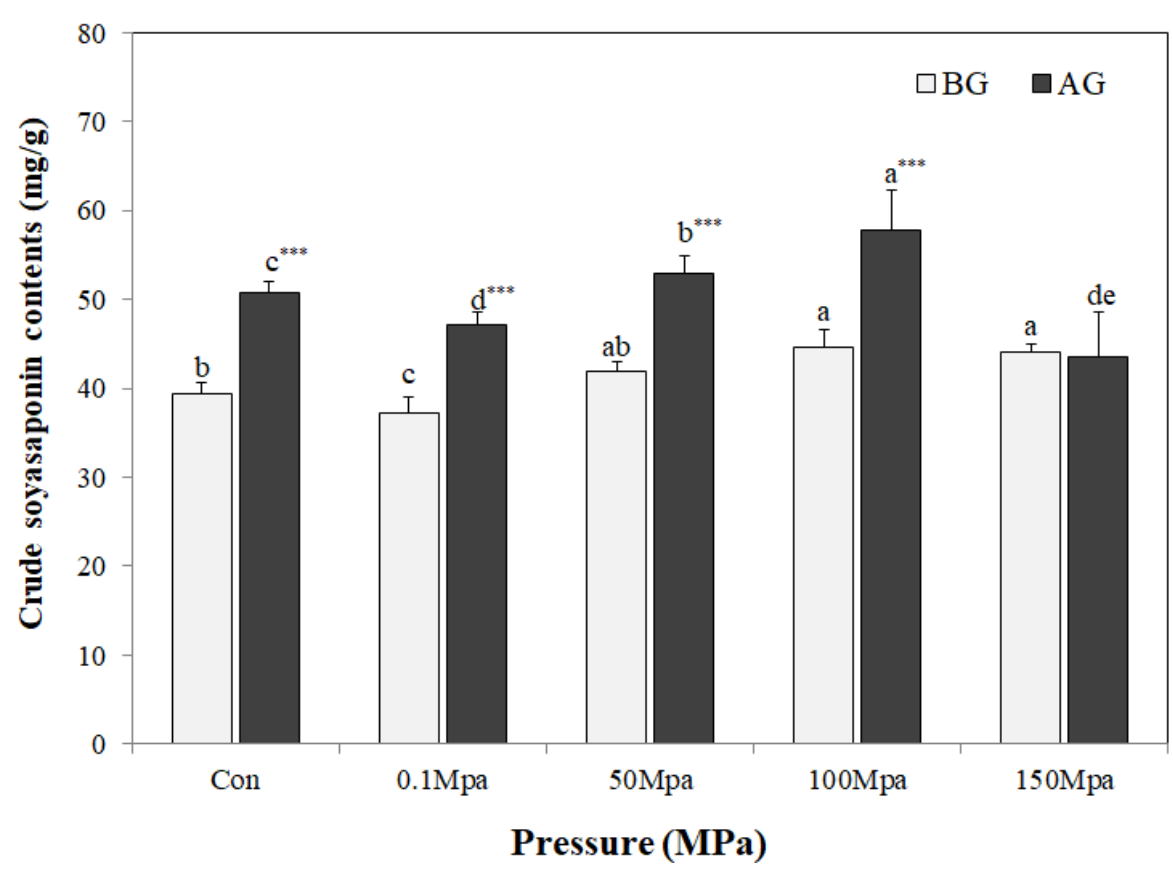

Figure 1. Changes in crude saponin contents with high hydrostatic pressure treatment of black soybean before(BG) and after germination(AG). Values are mean \pm SD of 3 replicates. Different small letters in the same items indicate a significant difference $(\mathrm{p}<0.05)$ among different pressure $(0.1-150 \mathrm{Mpa})$. ${ }^{*} \mathrm{p}<0.05 ;{ }^{* *} \mathrm{p}<0.01 ;{ }^{* * *} \mathrm{p}<0.001$; significantly different by Student's $\mathrm{t}$ test between before and after germination.

Also, The results of Figure 1 showed that HHP treatment contributed to improve production of soyasaponin in black soybean sprout, which varied in accordance with applied pressure. Crude soyasaponin contents of black soybean sprout increase progressively between 50.76 and 57.85 $\mathrm{mg} / \mathrm{g}$ as the applied pressure increases to $100 \mathrm{MPa}$, and then decrease to $43.54 \mathrm{mg} / \mathrm{g}$ with further increases in pressure. These results may be attributed to the stimulation of enzyme activity through the denaturation of substrates at pressures lower than $200 \mathrm{MPa}$; enzyme stimulation is known to play a major role in enzymatic hydrolysis [25]. Firstly, some of the bound soyasaponins are converted into extractable free soyasaponins during germination and HHP treatment as the cell walls are hydrolyzed, allowing increased solvent influx with increasing pressure. Secondly, the soyasaponin content is dictated by the enzyme-catalyzed biosynthesis and conversion of soyasaponins into essential secondary metabolites [23,24], and this enzyme activity is much higher during germination and HHP treatment [26].

\subsection{Soyasaponin Compositions}

The influence of germination and HHP treatment on the soyasaponin compositions, with respect to the A and B groups and the seven distinct forms of soyasaponin $A a$, $\mathrm{Ab}, \mathrm{Ba}, \mathrm{Bb}, \mathrm{Bc}$, soyasaponin A, and soyasapogenol B, in black soybean are represented in Table 1. The total soyasaponin content changes with germination and with applied pressure. The total soyasaponin contents of black soybean significantly increase from $111.44 \mathrm{mg} / 100 \mathrm{~g}$ to $153.32 \mathrm{mg} / 100 \mathrm{~g}$ after germination. In addition, the total soyasaponin contents in germinated black soybean treated by HHP range from 153.32 to $351.19 \mathrm{mg} / 100 \mathrm{~g}$. The highest total soyasaponin content $(351.19 \mathrm{mg} / 100 \mathrm{~g}$ ) is observed at $100 \mathrm{MPa}$ after germination. These results suggest that the total soyasaponin content is also affected synergistically by the combined effects of germination and
HHP treatment compared to germination or HHP treatment alone. Changes in the individual amounts of the seven distinct forms were monitored with combined germination and HHP treatment. Soyasaponin Aa was detected as a major A-group soyasaponin in the raw black soybean; the Aa contents are decreased with increasing applied pressure, although they are linearly increased from $1.14 \mathrm{mg} / 100 \mathrm{~g}$ to $3.86 \mathrm{mg} / 100 \mathrm{~g}$ with germination. Soyasaponin $\mathrm{Bb}$, a major B-group soyasaponin in raw black soybean, showed content increases in raw black soybean and germinated black soybean increase from 94.55 and $111.98 \mathrm{mg} / 100 \mathrm{~g}$ to 87.46 and $128.95 \mathrm{mg} / 100 \mathrm{~g}$, respectively, after HHP treatment at $100 \mathrm{MPa}$. The Bc content changes most drastically among the seven distinct forms. The $\mathrm{Bc}$ contents of raw black soybean and germinated black soybean increase from 9.91 and 25.85 $\mathrm{mg} / 100 \mathrm{~g}$ to 20.44 and $241.33 \mathrm{mg} / 100 \mathrm{~g}$, respectively, after HHP treatment at $150 \mathrm{MPa}$. These results are in close agreement with those reported by Shomoyamada and Okubo [27], which showed that the amounts of group A and Sg-6 saponins were reduced by 2.3- and 1.3-fold, respectively, while those of $B$

2.5 -fold relative to those of mature seeds. These findings show that the group A and Sg-6 saponins in mature seeds were degraded and/or translocated by germination, whereas B-group saponins were newly synthesized. Kurosawa et al. [23] reported the detection of uridine diphosphate (UDP)-glucuronic acid:soyasapogenol glucuronosyltransferase (UGASGT) activity for group B soyasaponin synthesis in microsomal fractions from germinating soybean seeds. These findings suggest that UGASGT was a specific enzyme for UDP-glucuronic acid as a donor and for soyasapogenols as acceptors, and that it was related to the biosynthesis of the sugar chain in soybean saponins. In addition, according to investigation of $\mathrm{Gu}$ et al. [28], B soyasaponins linked with 2,3-dihydro2,5-dihydroxy-6-methyl-4-pyrone (DDMP) soyasaponins, which are widely distributed in legumes as the most 
abundant group in soybeans, were also hydrolyzed to B soyasaponins and DDMP derivatives during sprouting. Thus, increases in B-group saponin levels are based on biosynthesis according to the activation of soyasapogenol glucuronosyltransferase, or to the conversion of B-group soyasaponin to DDMP soyasaponin. Therefore, the content of B-group soyasaponins is increased by germination and HHP treatment.

Table 1. Changes in soyasaponin compositions of black soybean before (BG) and after germination (AG) with high hydrostatic pressure treatment

\begin{tabular}{|c|c|c|c|c|c|c|c|c|c|c|c|c|}
\hline \multirow[b]{2}{*}{ 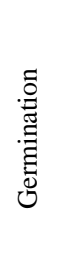 } & \multirow[b]{2}{*}{ 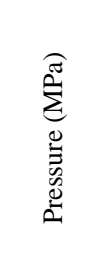 } & \multicolumn{11}{|c|}{ Contents of soyasaponin (mg/100 g) } \\
\hline & & 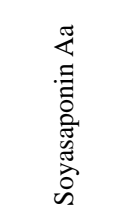 & 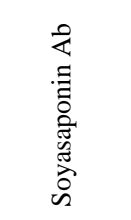 & 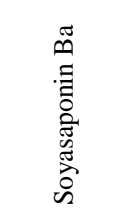 & 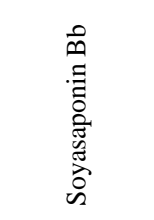 & 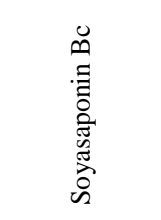 & 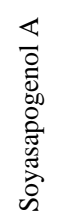 & 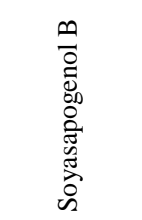 & 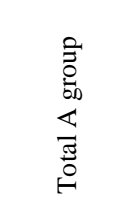 & 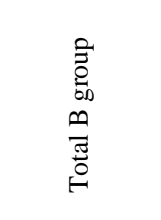 & 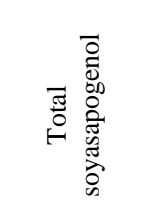 & Total \\
\hline \multirow{5}{*}{ BG } & Con & $1.14 \pm 0.04$ & $3.29 \pm 0.39$ & $1.18 \pm 0.62$ & $94.75 \pm 0.05$ & $9.91 \pm 0.29$ & ND & $1.19 \pm 0.13$ & $4.42 \pm 0.35$ & $105.83 \pm 0.39$ & $1.19 \pm 0.13$ & $111.44 \pm 0.09$ \\
\hline & $0.1 \mathrm{MPa}$ & $1.17 \pm 0.03$ & ND & $0.73 \pm 0.11$ & $90.11 \pm 0.82$ & $11.21 \pm 0.29$ & ND & $1.50 \pm 0.12$ & $1.17 \pm 0.03$ & $102.05 \pm 0.76$ & $1.50 \pm 0.12$ & $104.72 \pm 0.69$ \\
\hline & $50 \mathrm{MPa}$ & ND & ND & ND & $79.03 \pm 0.65$ & $12.69 \pm 0.21$ & ND & $2.55 \pm 0.05$ & ND & $91.72 \pm 0.86$ & $2.55 \pm 0.05$ & $94.27 \pm 0.81$ \\
\hline & $100 \mathrm{MPa}$ & ND & ND & ND & $87.46 \pm 0.86$ & $22.91 \pm 1.45$ & ND & $4.45 \pm 0.63$ & ND & $110.37 \pm 0.8$ & $4.45 \pm 0.63$ & $114.82 \pm 1.39$ \\
\hline & $150 \mathrm{MPa}$ & ND & ND & ND & $86.72 \pm 0.47$ & $20.44 \pm 0.01$ & ND & $6.05 \pm 1.03$ & ND & $107.16 \pm 0.48$ & $6.05 \pm 1.03$ & $113.21 \pm 0.55$ \\
\hline \multirow{5}{*}{ AG } & Con & $3.86 \pm 0.05$ & ND & ND & $111.98 \pm 1.39$ & $25.85 \pm 0.35$ & ND & $11.64 \pm 0.00$ & $3.86 \pm 0.05$ & $137.83 \pm 1.22$ & $11.64 \pm 0.00$ & $153.32 \pm 1.2$ \\
\hline & $0.1 \mathrm{MPa}$ & $4.78 \pm 0.14$ & ND & ND & $116.67 \pm 2.94$ & $47.82 \pm 1.48$ & ND & $11.08 \pm 0.48$ & $4.78 \pm 0.14$ & $164.49 \pm 3.33$ & $11.08 \pm 0.48$ & $180.34 \pm 2.97$ \\
\hline & $50 \mathrm{MPa}$ & ND & ND & ND & $108.96 \pm 6.03$ & $148.87 \pm 1.49$ & ND & $13.27 \pm 0.30$ & ND & $257.82 \pm 5.64$ & $13.27 \pm 0.30$ & $271.1 \pm 5.69$ \\
\hline & $100 \mathrm{MPa}$ & ND & ND & ND & $128.95 \pm 0.14$ & $202.9 \pm 2.46$ & ND & $18.47 \pm 0.00$ & ND & $331.84 \pm 2.36$ & $18.47 \pm 0.00$ & $350.31 \pm 2.36$ \\
\hline & $150 \mathrm{MPa}$ & ND & ND & ND & $87.75 \pm 2.28$ & $241.33 \pm 1.79$ & ND & $22.11 \pm 0.79$ & ND & $329.08 \pm 5.11$ & $22.11 \pm 0.79$ & $351.19 \pm 4.68$ \\
\hline
\end{tabular}

Values are mean \pm SD of 3 replicates. Different small letters in the same items indicate a significant difference $(\mathrm{p}<0.05)$ among different pressure (0.1-150Mpa). ${ }^{*} \mathrm{p}<0.05 ;{ }^{* *} \mathrm{p}<0.01 ;{ }^{* * *} \mathrm{p}<0.001$; significantly different by Student's t test between before and after germination.

25

$\square 25 \mu \mathrm{g} / \mathrm{mL} \quad \square 50 \mu \mathrm{g} / \mathrm{mL} \quad \square 100 \mu \mathrm{g} / \mathrm{mL} \quad \square 200 \mu \mathrm{g} / \mathrm{mL}$

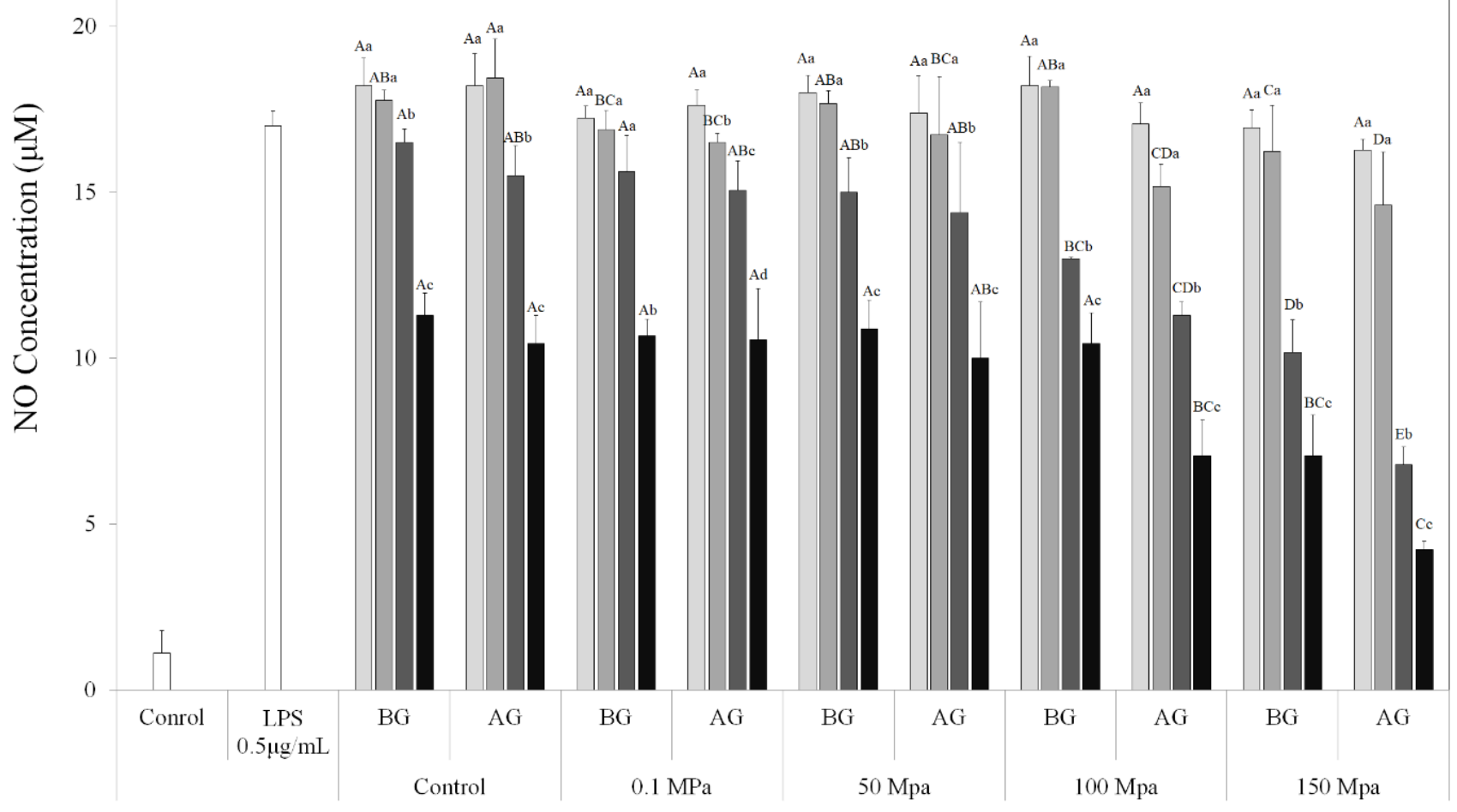

Pressure(Mpa)-Germination period(day)

Figure 2. Effect of crude saponin extracts of black soybean treated by high hydrostatic pressure treatments and before(BG) and after germination(AG) on nitrate oxide concentration of RAW 264.7 cell stimulated with LPS $(0.5 \mu \mathrm{g} / \mathrm{mL})$ Values are mean \pm SD of 3 replicates. Different capital letters in the same items indicate a significant difference $(\mathrm{p}<0.05)$ among different pressure $(0.1-150 \mathrm{Mpa})$. Different small letters in the same items indicate a significant difference $(\mathrm{p}<0.05)$ among different germination periods of black soybean. 


\subsection{Inhibition of NO Production in RAW 264.7 Cell}

The anti-inflammatory activities of crude soyasaponin extracts, including their ability to inhibit $\mathrm{NO}$ and pro-inflammatory cytokine (TNF- $\alpha$, IL-1 $\beta$, and IL-6) production, were investigated. The cytotoxic activity of the samples was determined using RAW 264.7 cells before the evaluation of anti-inflammatory effects. Cell treatments with extracts in less than $200 \mu \mathrm{g} / \mathrm{mL}$ concentrations did not affect RAW 264.7 cell viability (data not shown). The effects of crude soyasaponin extracts from black soybeans treated with HHP after germination on LPS-induced NO were investigated by treating RAW 264.7 cells with various concentrations of extracts and $0.5 \mu \mathrm{g} / \mathrm{mL}$ LPS for $24 \mathrm{~h}$. The influences of germination and HHP treatment on soyasaponin extract-induced inhibition of NO production are shown in Figure 2. The NO concentration in the medium is markedly increased after treatment with $0.5 \mu \mathrm{g} / \mathrm{mL}$ LPS for $24 \mathrm{~h}(16.99 \mu \mathrm{M})$ compared to the concentration in the medium of the unstimulated control $(1.12 \mu \mathrm{M})$. Most of the crude soyasaponin extracts inhibit NO production in a dose-dependent manner at concentrations $>25 \mu \mathrm{g} / \mathrm{mL}$ $(p<0.05)$. The inhibitory effects of crude soyasaponin extracts on NO synthesis, an indicator of their anti-inflammatory potential, by LPS-stimulated macrophages were thus enhanced by HHP treatment after germination. The NO concentration in the medium of RAW 264.7 cells is slightly decreased from $11.28 \mu \mathrm{M}$ to $10.44 \mu \mathrm{M}$ in the presence of $200 \mu \mathrm{g} / \mathrm{mL}$ extracts from raw or germinated black soybean. Furthermore, the NO concentrations in the media of RAW 264.7 cells treated with $200 \mu \mathrm{g} / \mathrm{mL}$ crude soyasaponin extracts from germinated black soybean subjected to $150 \mathrm{MPa}$ for $24 \mathrm{~h}$ ranged from 4.22 to $10.44 \mu \mathrm{M}$. The strongest inhibitory effect (NO concentration of $4.22 \mu \mathrm{M})$ is observed after treatment with crude soyasaponin extracts subjected to germination and $150 \mathrm{MPa}$ for $24 \mathrm{~h}$. The crude soyasaponin extract is shown to contain high amounts of soyasaponin B and soyasapogenol B, as well as an increase in the peak area of a peak corresponding to an unknown component in the HPLC analysis of crude soyasaponin extracts at $205 \mathrm{~nm}$. Among soyasaponins and soyasapogenols, the anti-inflammatory characteristics of soyasaponin $\mathrm{Ab}, \mathrm{Ba}$, $\mathrm{Bb}, \mathrm{Bc}$ and soyasapogenol $\mathrm{B}$ include the inhibition of TNF- $\alpha$, IL-1 $\beta$, PGE2, NO, COX-2, and iNOS productions, I $\kappa$ B $-\alpha$ phosphorylation, NF- $\kappa \mathrm{B}$ activity, and iNOS enzyme activity in LPS-stimulated macrophages [29,30].

\subsection{Inhibition of Pro-inflammatory Cytokine Production}

TNF- $\alpha$, IL- $1 \beta$ and IL-6 are important pro-inflammatory cytokines linked to the pathogenesis of many infectious and inflammatory diseases, including cancer [31]. As shown in Figure 3, LPS-stimulated RAW 264.7 cells markedly up-regulated TNF- $\alpha$, IL-1 $\beta$, and IL-6 production. The concentrations of TNF- $\alpha$, IL- $1 \beta$, and IL-6 in the media of untreated cells (7.01 ng/mL, not detected, and not detected, respectively) are increased after treatment with LPS $(0.5 \mu \mathrm{g} / \mathrm{mL})$ for $24 \mathrm{~h}$ to $635.17,111.00$, and $1.14 \mathrm{ng} / \mathrm{mL}$, respectively. The LPS-induced increases in cytokine concentrations are inhibited in a dose-dependent manner by $>50 \mu \mathrm{g} / \mathrm{mL}$ crude soyasaponin extracts from germinated black soybean treated at $150 \mathrm{MPa}$. Crude soyasaponin extracts from raw black soybean slightly inhibit the concentrations of TNF- $\alpha$, IL- $1 \beta$, and IL-6 in RAW 264.7 culture supernatants, compared to the concentrations in positive control LPS-stimulated supernatants. HHP treatment after germination enhances the pro-inflammatory cytokine inhibition behavior; treatment of LPS-stimulated RAW 264.7 cells with the highest concentration $(200 \mu \mathrm{g} / \mathrm{mL})$ of soyasaponin extracted from germinated black soybean subjected to 150 MPa causes reductions in TNF- $\alpha$, IL- $1 \beta$, and IL-6 levels to $329.67,34.33$ and $0.73 \mathrm{ng} / \mathrm{mL}$, respectively. Kang et al. [32] reported that crude extract of soyasaponins significantly inhibited the production of pro-inflammatory cytokine TNF- $\alpha$ and chemokine MCP-1, the inflammatory mediator's prostaglandin E2 and nitric oxide, and the inflammatory enzymes cyclooxygenase (COX)-2 and inducible nitric oxide synthase (iNOS) and degradation of I $\kappa \mathrm{B}-\alpha$, an inhibitor of $\mathrm{NF}-\kappa \mathrm{B}$, in lipopolysaccharide (LPS)-stimulated macrophages. Therefore, these results confirm that the crude soyasaponin extract of germinated black soybean show anti-inflammatory effects that HHP treatment improves. The enhanced production of soyasaponin B, soyasapogenol B, and the unknown soyasaponin, shown in HPLC, and the conversion of soyasaponin may positively affect the anti-inflammatory activity of soyasaponin extracts. Further study is required to isolate and identify the structure of active soyasaponin to clarify this correlation between soyasaponin conversion and anti-inflammatory characteristics.

\subsection{Anti-obesity Activities in 3T3-L1 Adipocyte Cell}

The cytotoxic activity of samples was determined using 3T3-L1 cells before the evaluation of lipid accumulation during 3T3-L1 adipocyte differentiation. Cell treatments with extracts of $<400 \mu \mathrm{g} / \mathrm{mL}$ concentrations did not affect 3T3-L1 cell viability (data not shown). 3T3-L1 pre-adipocytes are among the best-characterized and most reliable in vitro models in research on new health benefit foods and agents for obesity or weight control in many studies [33]. The suppression of lipid accumulation during 3T3-L1 adipocyte differentiation has been reported as an indicator of an anti-adipogenic effect [34]. The suppression effects of lipid accumulation by crude soyasaponin extracts from black soybean treated with HHP after germination are presented in Figure 4 and Figure 5. Cellular lipid accumulation and the lipid droplets are more thoroughly suppressed by the 150-MPa HHP-treated crude soyasaponin extracts from germinated black soybean than by raw black soybean, in a dose-dependent manner. The lipid accumulation rate (\% positive control) during 3T3-L1 adipocyte differentiation in germinated black soybean treated by HHP ranges from 93.25 to $65.95 \%$ at the concentration of $400 \mu \mathrm{g} / \mathrm{mL}$. The highest anti-obesity effect is observed after germination and HHP treatment at $150 \mathrm{MPa}$. Plant saponins have been reported to reduce obesity by restricting calorie intake, inhibiting pancreatic lipase, and modulating certain genes involved in lipid metabolism [35,36]. Reports on the 
effect of soyasaponins A and B in high fat-fed mice are rare; only total soyasaponin extracts or soyasaponin B have been studied for their hypolipidemic and hypocholesterolemic effects [37]. However, it was previously shown that soyasaponin extracts show dose-dependent inhibition of the accumulation of lipids in 3T3-L1 adipocytes [38]. Therefore, these results suggest that the
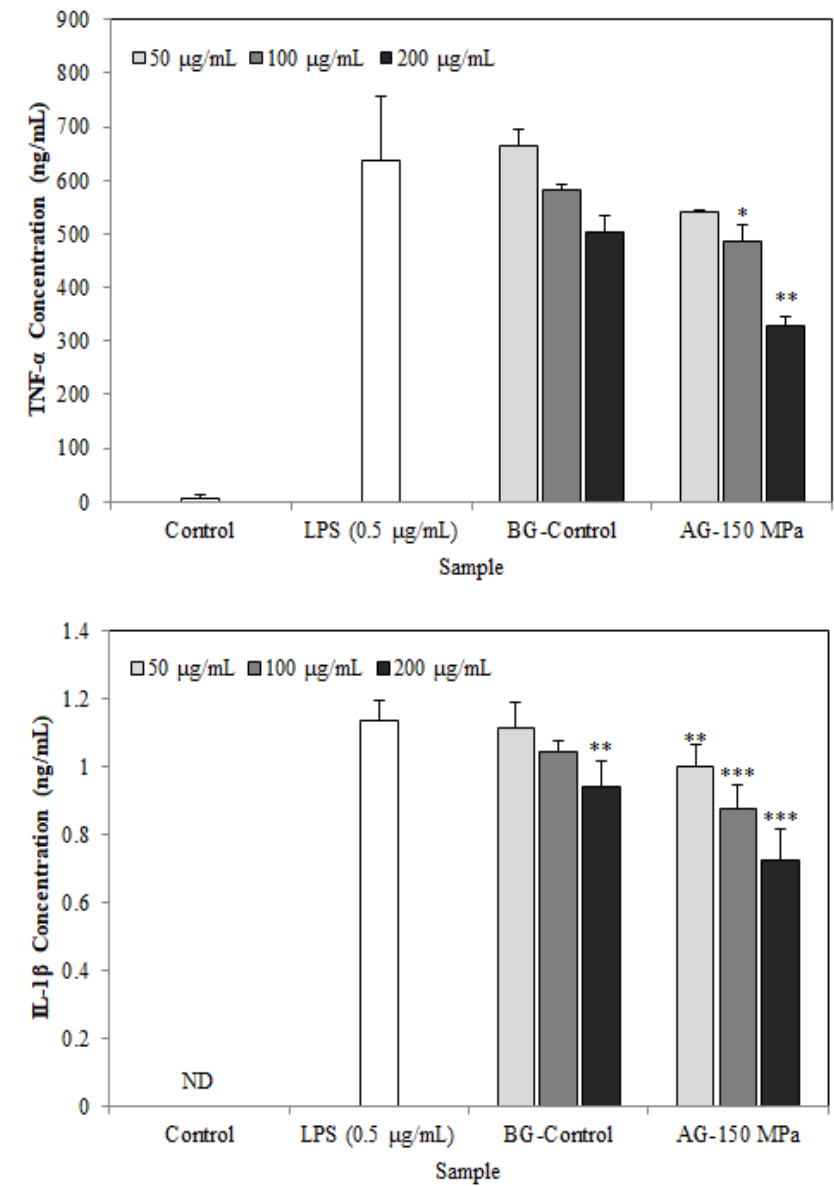

crude soyasaponin extract of germinated black soybean has an anti-obesity effect that HHP treatment improves. The enhanced production of soyasaponin B, soyasapogenol $B$, and the unknown soyasaponin component, as well as the conversion of soyasaponin, may positively affect lipid accumulation during 3T3-L1 adipocyte differentiation.

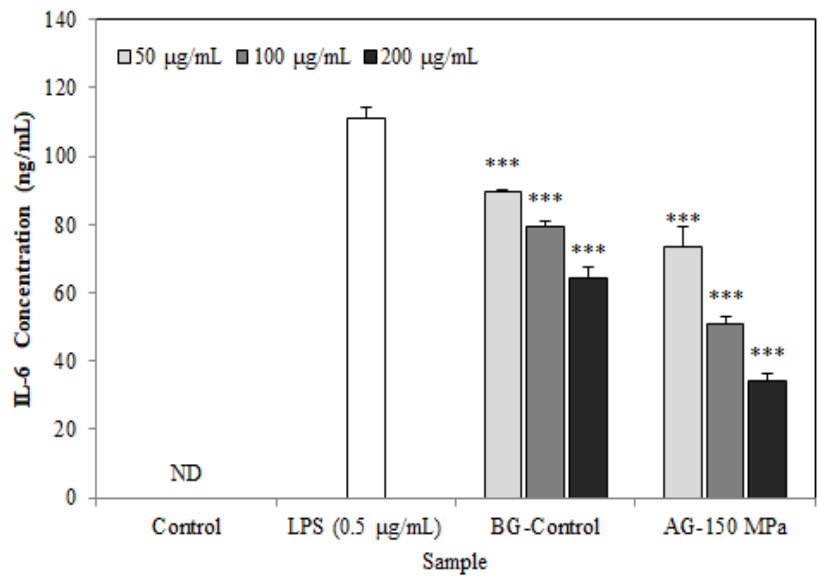

Figure 3. Effect of crude soyasaponin extracts of black soybean treated by high hydrostatic pressure treatments and before(BG) and after germination(AG) on TNF- $\alpha$, IL-6 and IL-1 $\beta$ secretion of RAW 264.7 cell stimulated with LPS $(0.5 \mu \mathrm{g} / \mathrm{mL}) . * \mathrm{p}<0.05, * * \mathrm{p}<0.01,{ }^{* * *} \mathrm{p}<0.001$; Significantly different by paired t-test, significantly different by Student's t-test between control stimulated with LPS $(0.5 \mu \mathrm{g} / \mathrm{mL})$ and samples.
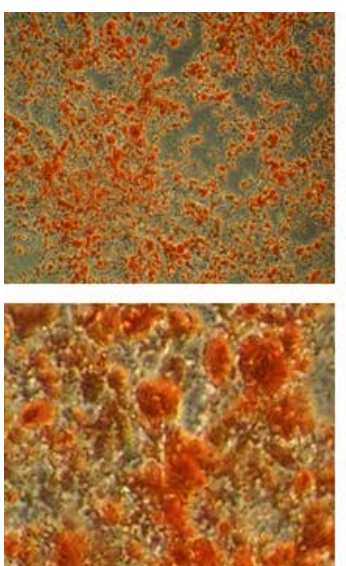

Control
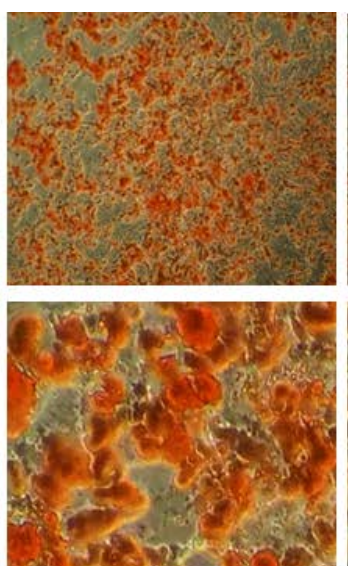

100
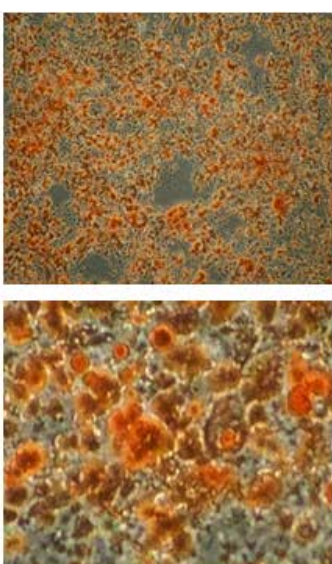

400
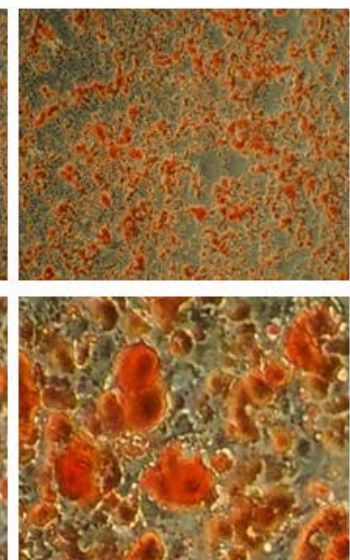

100
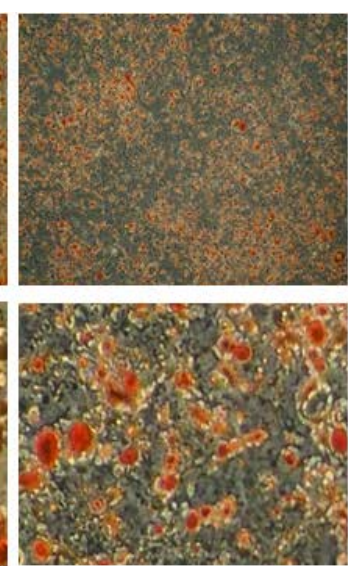

400

soyasaponin extract $A(\mu \mathrm{g} / \mathrm{mL})$

soyasaponin extract $B(\mu \mathrm{g} / \mathrm{mL})$

Figure 4. Effect of soyasaponin extracts of soybean treated by high hydrostatic pressure treatments and germination on lipid accumulation in differentiated 3T3-L1 adipocytes. (A: before germination, B: $150 \mathrm{MPa}$ after germination). Cells were treated with DGSE for 4 days (days $0 \sim 4$ ) during differentiation. Lipid content was measured on day 8 by Oil-O-Red staining method. Assays were performed in triplicates for each treatment 


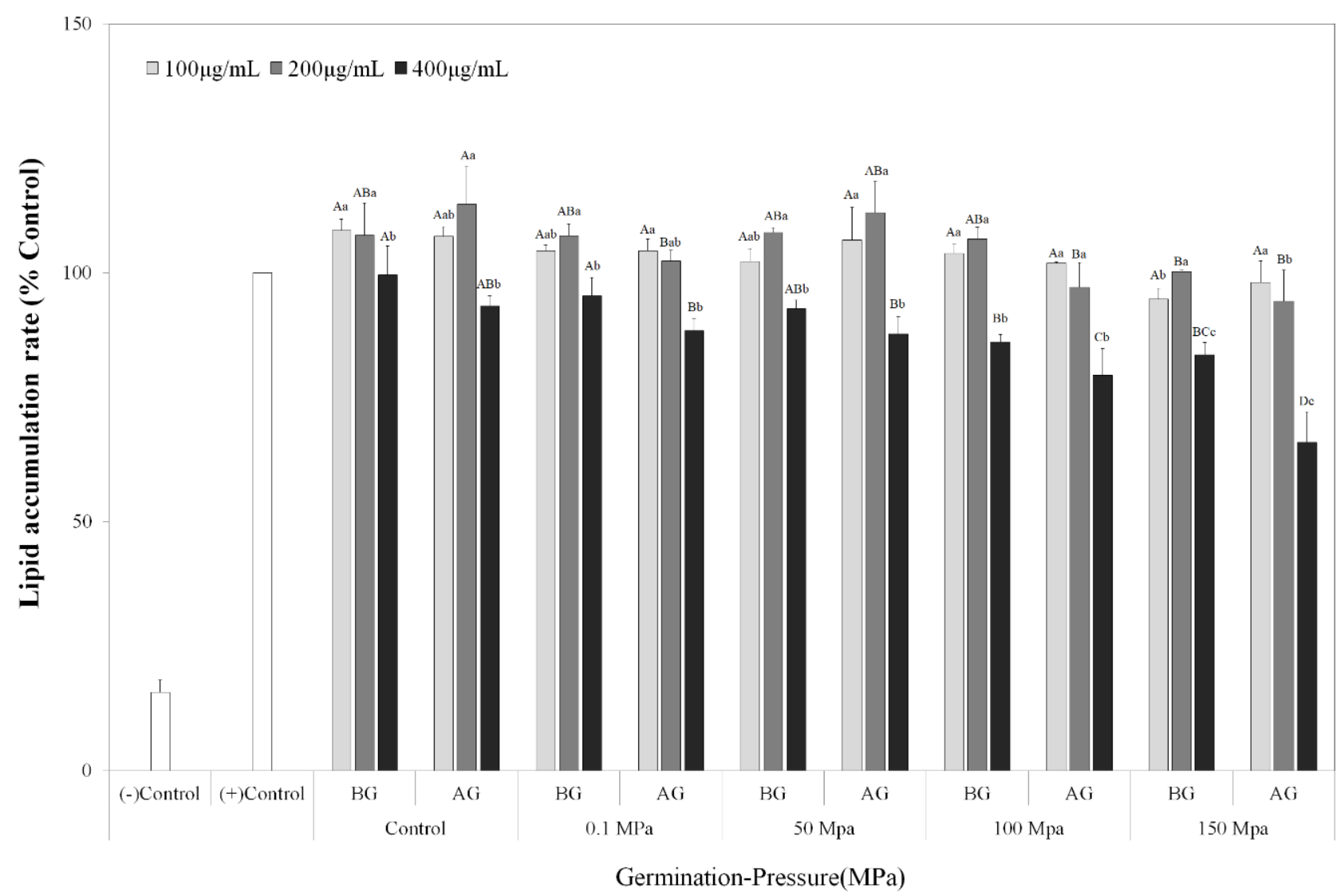

Figure 5. Effect of soyasaponin extracts of soybean treated by high hydrostatic pressure treatments and before(BG) and after germination(AG) on lipid accumulation in differentiated 3T3-L1 adipocytes. Cells were treated with DGSE for 4 days (0 4 days) during differentiation. Assays were performed in triplicates for each treatment. Values are mean $\pm S D$ of 3 replicates. Different capital letters in the same items indicate a significant difference $(p<0.05)$ among different pressure (0.1-150Mpa). Different small letters in the same items indicate a significant difference $(\mathrm{p}<0.05)$ among different germination periods of black soybean

\subsection{Conclusion}

The beneficial effects of soyasaponins in the amelioration of chronic diseases have been linked to their modulation of inflammation and obesity, and single processing steps of germination and HHP treatment have shown efficacy in increasing the functional properties of cereals and legumes. Thus, the combination of HHP treatment and germination may enhance the functionalities of soyasaponins extracted from black soybean. Indeed, combined treatment by HHP and germination yielded crude soyasaponin extracts with greater anti-inflammatory and anti-obesity properties. The highest crude soyasaponin contents were measured in black soybean subjected to 100 $\mathrm{MPa}$ for $24 \mathrm{~h}$ after germination. Soyasaponin $\mathrm{Bb}$ and $\mathrm{Bc}$ contents in raw black soybean and germinated black soybean were increased after HHP treatment at $100 \mathrm{MPa}$ and $150 \mathrm{MPa}$. The highest anti-obesity effect was observed after 24 h 150-MPa HHP treatment after germination. Regarding anti-inflammatory activity, germinated soybean extracts treated at $150 \mathrm{MPa}$ significantly inhibited the LPS-induced expression of inflammatory markers TNF- $\alpha$, IL-1 $\beta$, and IL-6 by RAW 264.7 macrophages. This study provides valuable information on the application of HHP in combination with pre-germination for improving the utilization of soyasaponin extracts as anti-inflammatory and anti-obesity agents. Further study is required to investigate the development of a high-pressure, short-time pressure and enzyme treatment method to resolve practical limits of HHP, after the identification and isolation of marker compounds from germinated, HHPtreated black soybean saponin extracts.

\section{Acknowledgements}

This research was supported by the High Value added Food Technology Development Program (316052-03), Ministry of Agriculture, Food and Rural Affairs

\section{Conflict of Interest}

The authors have no conflicts of interest to declare.

\section{References}

[1] Ireland, P.A., Dziedzic, S.Z. and Kearsley, M.W, "Saponin content of soya and some commercial soya products by means of high-performance liquid chromatography of the sapogenins”, J Sci Food Agric, 37 (7). 694-698. July 1986.

[2] Yoshikoshi, M., Yoshiki, Y., Okubo, K., Seto, J. and Sasaki, Y., "Prevention of hydrogen peroxide damage by soybean saponins to mouse fibroblasts", Planta, 62(3). 252-255. Jun 1996.

[3] Ishii, Y. and Tanizawa, H., "Effects of soyasaponins on lipid peroxidation through the secretion of thyroid hormones", Biol Pharm Bull, 29(8). 1759-1763. Aug 2006.

[4] Lee, S. O., Simons, A. L., Murphy, P. A. and Hendrich, S., "Soyasaponins lowered plasma cholesterol and increased fecal bile acids in female golden Syrian hamsters” Exp Biol Med, 230(7). 472-478. July 2005. 
[5] Takahashi, S., Hori, K., Shinbo, M., Hiwatashi, K., Gotoh, T. and Yamada, S., "Isolation of human renin inhibitor from soybean: soyasaponin I is the novel human renin inhibitor in soybean", Biosci Biotechnol Biochem, 72(12). 3232-3236. Dec 2008.

[6] Kinjo, J., Imagire, M., Udayama, M., Arao, T. and Nohara, T., "Structure-hepatoprotective relationships study of soyasaponins IIV having soyasapogenol B as aglycone”, Planta medica, 64(3). 233-236. Apr 1998.

[7] Kerwin, S. M., "Soy saponins and the anticancer effects of soybeans and soy-based foods", Curr Med Chem Anticancer Agents, 4(3). 263-272. May 2004.

[8] Marathe, S. A., Rajalakshmi, V., Jamdar, S.N. and Sharma, A. "Comparative study on antioxidant activity of different varieties of commonly consumed legumes in India”, Food Chem Toxicol, 49(9). 2005-2012. May 2011.

[9] Guajardo-Flores, D., Serna-Saldívar, S.O. and Gutiérrez-Uribe, J.A., "Evaluation of the antioxidant and antiproliferative activities of extracted saponins and flavonols from germinated black beans (Phaseolus vulgaris L.)”, Food Chem, 141(2). 1497-1503. Nov 2013.

[10] Liu, C., Li, S., Tsao, R., Li, S. and Zhang, Y., "Extraction and isolation of potential anti-stroke compounds from black soybean (Glycine max L. Merrill) guided by in vitro PC12 cell model”, J Funct Foods, 31. 295-303. Apr 2017.

[11] Mbithi, S., Van Camp, J., Rodriguez, R. and Huyghebaert, A., "Effects of sprouting on nutrient and antinutrient composition of kidney beans (Phaseolus vulgaris var. Rose coco)”, Eur Food Res Technol, 212(2). 188-191. Jan 2001.

[12] Paucar-Menacho, L.M., Berhow, M.A., Mandarino, J.M.G., Chang, Y.K. and de Mejia, E.G., "Effect of time and temperature on bioactive compounds in germinated Brazilian soybean cultivar BRS 258”, Food Res Int, 43(7). 1856-1865. Aug 2010.

[13] Bau, H.M., Villaume, C., and Mejean, L., "Effects of soybean (Glycine max) germination on biologically active components, nutritional values of seeds, and biological characteristics in rats", Nahrung-Food, 44(1). 2-6. Feb 2000.

[14] Lopes, M.L., Valente Mesquita, V.L., Chiaradia, A.C., Fernandes, A.A. and Fernandes, P.M., High hydrostatic pressure processing of tropical fruits. Ann NY Acad Sci, 1189. 6-15. Feb 2010.

[15] Mozhaev, V.V., Heremans, K., Frank, J., Mansson, P. and Balny, C., "High pressure effects on protein structure and function", Proteins, 24. 81-91. Jan 1996.

[16] Kim, M.Y., Jang, G.Y., Oh, N.S., Baek, S.Y., Lee, S.H., Kim, K.M., Kim, T.M., Lee, J.S., Jeong, H.S., "Characteristics and in vitro anti-inflammatory activities of protein extracts from pre-germinated black soyneam [Glycine max (L.)] treated with high hydrostatic pressure”, Innov Food Sci Emerg Technol, 43. 84-91. Oct 2017.

[17] Ana, L., Tarek, E., Montserrat, D., Teresa, O., Isabel, E., Teresa, H., Pilar, G., Olga, P. and Emiliak, C., "Effect of cooking and germination on phenolic composition and biological properties of dark beans (Phaseolus vulgaris L.)”, Food Chem, 138. 547-555. May 2013.

[18] Berhow, M. A., Cantrell, C.L., Duval, S.M., Dobbins, T.A Maynes, J. and Vaughn, S.F., "Analysis and quantitative determination of group B saponins in processed soybean products”, Phytochem Anal, 13(6). 343-348. Nov 2002.

[19] Hubert, J., Berger, M., Daydé, J., "Use of a simplified HPLC- UV analysis for soyasaponin B determination: study of saponin and isoflavone variability in soybean cultivars and soy-based health food”, J Agric Food Chem, 53(10). 3923-3930. Apr 2005.

[20] Ishiyama, M., Tominaga, H., Shiga, M., Sasamoto, K., Ohkura, Y., Ueno, K., "A combined assay of cell viability and in vitro cytotoxicity with a highly water-soluble tetrazolium salt, neutral red and crystal violet”, Biol Pharm Bull, 19. 1518-1520. Nov 1996.
[21] Srisook, K., Han, S.S, Choi, H.S., Li, M.H., Ueda, H., Kim, C., Cha, Y.N., "CO from enhanced HO activity or from CORM-2 inhibits both $\mathrm{O} 2-$ and $\mathrm{NO}$ production and downregulates $\mathrm{HO}-1$ expression in LPS-stimulated macrophages”, Biochem Pharmacol, 71(3). 307-318. Jan 2006.

[22] Chang, S.Y., Han, S., Changes of soyasaponin contents in soybean sprouts. Korean J Crop Sci, 61(1). 57-63. Jan 2016.

[23] Kurosawa, Y., Takahara, H., Shiraiwa, M., "UDP-glucuronic acid soyasapogenol glucuronosyltransferase involved in saponin biosynthesis in germinating soybean seeds” Planta, 215(4). 620-629. Aug 2002.

[24] Yoshiki, Y., Kudou, S., OKuBo, K., "Relationship between chemical structures and biological activities of triterpenoid saponins from soybean” Biosci Biotechnol Biochem, 62(12). 2291-2299. May 2014

[25] Cheftel, J.C., "Review: High-pressure, microbial inactivation and food preservation” Food Sci Technol Int, 1. 75-90. Aug 1995

[26] Gross, G.G., "Biosynthesis and metabolism of phenolic acids and monolignols. In Takayoshi Higuchi (eds), Biosynthesis and biodegradation of wood components” Elsevier, pp. 229-271.

[27] Shimoyamada, M., Okubo, K., "Variation in saponin contents in germinating soybean seeds and effect of light irradiation” Agric Biol Chem, 55(2), 577-579. Sep 2014.

[28] Gu, E.J., Kim, D.W., Jang, G.J., Song, S.H., Lee, J.I., Lee, S.B., Kim, H.J., "Mass-based metabolomic analysis of soybean sprouts during germination” Food chem, 217, 311-319. Feb 2017.

[29] Zha, L.Y., Mao, L.M., Lu, X.C., Deng, H., Ye, J.F., Chu, X.W., Luo, H.J., "Anti-inflammatory effect of soyasaponins through suppressing nitric oxide production in LPS-stimulated RAW 264.7

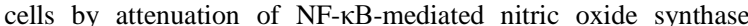
expression” Bioorg Med Chem Lett, 21(8), 2415-2418. Apr 2018.

[30] Lee, I.A., Park, Y.J., Yeo, H.K., Han, M.J., Kim, D.H. "Soyasaponin I attenuates TNBS-induced colitis in mice by inhibiting NF-кB pathway" J Agric Food Chem, 58(20), 10929-10934. Oct 2010

[31] Bertazza, L., Mocellin, S., “Tumor necrosis factor (TNF) biology and cell death” Front Biosci, 13, 2736-2743. Jan 2008.

[32] Kang, J.H., Sung, M.K., Kawada, T., Yoo, H., Kim, Y.K., Kim, J.S., Yu, R., "Soybean saponins suppress the release of proinflammatory mediators by LPS-stimulated peritoneal macrophages” Cancer let, 230(2), 219-227. Dec 2005.

[33] Kong, C.S., Kim, J.A., Kim, S.K., “Anti-obesity effect of sulfated glucosamine by AMPK signal pathway in 3T3-L1 adipocytes" Food Chem Toxicol, 47, 2401-2406. Oct 2009.

[34] Ghosh, C., Chung, H.Y., Nandre, R.M., Lee, J.H., Jeon, T.I., Kim, I.S., Yang, S.H., Hwang, S.G., “An active extract of Ulmus pumila inhibits adipogenesis through regulation of cell cycle progression in 3T3-L1 cells” Food Chem Toxicol, 50, 2009-2015. Jun 2012.

[35] Han, L.K., Kimura, Y., Kawashima, M., Takaku, T., Taniyama, T., Hayashi, T., Zheng, Y.N., Okuda, H., "Anti-obesity effects in rodents of dietary teasaponin, a lipase inhibitor” Int J Obes, 25, 1459-1464. Oct 2001

[36] Sunyoon, J., Mak-Soon, L., Yoonjin, S., Chong-Tai, K., In-Hwan, K., Young, S., Yangha, K., "Anti-obesity and antiinflammatory effects of high hydrostatic pressure extracts of ginseng in high-fat diet induced obese rats” J Funct Foods, 10, 167-177. Sep 2014.

[37] James, D.S., Satchithanandam, E., Joseph, S., Sun-Ok, L., "Effects of Group B Soyasaponins and soyasapogenol B on plasma cholesterol levels in golden gyrian hamsters” Adv Nutr Food Technol, 1(1-104), 1-8. Jan 2014.

[38] Seung, H.Y., Eun-Kyung, A., Jung, A.L., Tai-Sun, S., Chigen, T., Joowon, S., "Itabashi M and Gyuhwa C. (2015) Soyasaponins Aa and $\mathrm{Ab}$ exert an anti-obesity effect in 3T3-L1 adipocytes through downregulation of PPAR $\gamma$ " Phytother Res, 29, 281-287. Nov 2014 